\title{
Showcase EUROGRID - towards a European resource for gridded climate data, products and services
}

\author{
T. Klein and C. Persson \\ Swedish Meteorological and Hydrological Institute, Norrköping, Sweden \\ Received: 19 December 2008 - Revised: 27 February 2009 - Accepted: 6 April 2009 - Published: 29 April 2009
}

\begin{abstract}
EUROGRID is the vision of the European network of meteorological services (EUMETNET) about a European service for gridded climate monitoring information and products responding to the needs of users from a variety of domains. The EUMETNET project Showcase EUROGRID (S-EUROGRID) presented in this paper has the main objective to provide a better understanding of the EUROGRID idea and the way forward towards its realization. In addition to the identification of user requirements on gridded climate monitoring data and services S-EUROGRID aims to demonstrate example products and services building on existing shared gridded climate monitoring information available from European meteorological and hydrological services and other initiatives. The latter includes a harmonized visualization of data, the generation of example products/services and the dissemination of raw or processed data to the user community. For these purposes S-EUROGRID needed a simple and low-cost technical solution allowing for the seamless integration and dissemination of maps and data contributed by distributed resources, leading to Open Geospatial Consortium (OGC)-protocols as one promising option. In this concept, data providers can set up their own OGCcompliant services for climate data, which then can be viewed/accessed in a harmonized way, e.g. through the S-EUROGRID portal or by a user's specific client software (e.g. a GIS). Dynamic (i.e. real-time) generation of products is beyond the scope of S-EUROGRID, but the architectural choice of OGC-standards offers easy ways for client-side data processing, e.g., using the end user's GIS capacity. In addition, S-EUROGRID hosts a selection of pre-processed example products, illustrating the potential of combining gridded climate monitoring information with data and information sources from other thematic domains.
\end{abstract}

\section{Introduction}

EUROGRID is the idea about a European information resource based on gridded climate monitoring data and was born in the European network of meteorological services (EUMETNET). The EUROGRID service could provide information content and products to serve a variety of users from fields such as climatology, meteorology, environment, hydrology and oceanography, energy, agriculture, forestry, health and insurance. Conceptually, this idea integrates with European initiatives such as the EU's Global Monitoring for Environment and Security (GMES) and the Shared Environmental Information System (SEIS) proposed by the European Commission and with global initiatives such as the Global Earth Observation System of Systems (GEOSS) and the World Meteorological Organization (WMO) information system (WIS). Thematically and with regard to information content, there will be synergies between the EUROGRID idea and other projects on gridded climate monitoring information for Europe, such as the FP6 project ENSEMBLES or potential European re-analysis projects. On a technical level, the EUROGRID service's underlying architecture should be in line with the INSPIRE directive's requirements, allow for standardized dissemination of information and data and, thus, for interoperability with other services and a seamless integration with the user community.

As a first step towards the realization of the ambitious EUROGRID idea, EUMETNET established the Showcase EUROGRID (S-EUROGRID), a two year EUMETNET optional programme under the umbrella of the European Climate Support Network (ECSN). The S-EUROGRID network of partners is shown in Fig. 1.

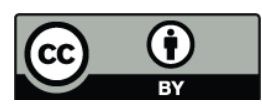

Correspondence to: T. Klein

(thomas.klein@smhi.se) 


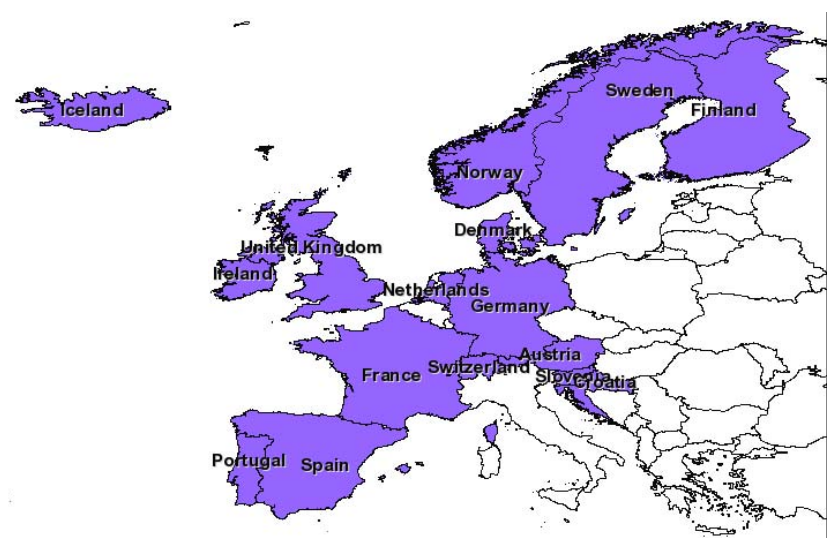

Figure 1. S-EUROGRID Consortium of partners in 2008: Austria (Austria Central Institute for Meteorology and Geodynamics), Croatia (Croatian Meteorological and Hydrological Service), Denmark (Danish Meteorological Institute), Finland (Finnish Meteorological Institute), France (Meteo France), Germany (Deutscher Wetterdienst), Iceland (Icelandic Meteorological Office), Ireland (Irish National Meteorological Service), Netherlands (Royal Meteorological Institute of the Netherlands), Norway (The Norwegian Meteorological Institute), Portugal (Instituto de Meteorologia), Slovenia (Environmental Agency of the Republic of Slovenia), Spain (Instituto Nacional de Meteorología, Sweden (Swedish Meteorological and Hydrological Institute), Switzerland (MeteoSwiss), United Kingdom (Met Office).

\section{The Showcase EUROGRID demonstration system}

\subsection{Example data}

For reasons of feasibility and due to budget constraints, the project works with example data provided in-kind (i.e. without funding/charging for costs) by the participants. The example data are inhomogeneous in many respects, e.g. temporal and spatial resolution, aggregation methods and in the techniques used for their production. In general, the gridding techniques applied for the production of S-EUROGRID datasets can be broadly divided into two categories:

- Interpolation of in-situ observations: The accuracy of the obtained results is strongly dependent on the density of the observation stations and measurements. Being independent of dynamic model formulations, these gridded data also provide a valuable resource for the validation/calibration of atmospheric models.

- Re-analysis based on data assimilation in a threedimensional dynamical weather prediction model: This approach combines in-situ and remote sensing measurements of different variables in a consistent way. Further refinements can be obtained through nested re-analysis (also referred to as dynamical downscaling) and/or twodimensional downscaling, using output from a coarser reanalysis as background information and - optionally
- assimilating additional streams of observations (e.g. other in-situ data, radar data).

Datasets used in S-EUROGRID comprise both national data sets with spatial resolutions on the order of a few $\mathrm{km}$ and pan-European datasets with grid spacings on the order of tens of km. A more detailed description of the datasets is beyond the scope of this short note. For an elaborate documentation of datasets and gridding techniques the interested reader is thus referred to the project's final report (http://www.e-grid.eu/public/FinalReport/). The diversity in national interpolation methods, spatio-temporal resolutions, aggregation periods and naming/time-stamping conventions is a well-known issue, e.g. from experiences within the COST Action 719 (http://www.knmi.nl/samenw/cost719/), and will need to be addressed in a follow-up project.

\subsection{Technical architecture}

For the visualization and dissemination of its example data and products, S-EUROGRID needed a flexible, robust and low-cost solution. With the vision of a mature European service integrating with a variety of systems, applications and user communities in mind, the choice of open and internationally and community-accepted standards was given, leading to the build-up of the S-EUROGRID solution around Open Geospatial Consortium (OGC, 2008) standards. This architectural choice ensures the seamless integration of distributed information resources and, in addition, a seamless integration of the information content with the GIS environments applied within the user communities. Maps and data from distributed service providers are linked together in a front-end application using an OpenLayers (OpenLayers, 2008) map service. For a harmonized visualization of the datasets and the dissemination of maps of essential climate variables (ECVs) the OGC Web Map Service (WMS) protocol is used. Data dissemination to test users is available through the OGC Web Coverage Service (WCS) protocol, allowing for easy usage of the data in a GIS and thus further client-side processing of the data. A mature national climate monitoring service built in this concept is already operated by an S-EUROGRID partner (www.senorge.no).

Parallel to this solution which serves the delivery of maps and raster data, a centralized MySQL database was set up for the provision of example time series at given locations. In principle, it would be desirable to have both time series at fixed locations and maps and data at fixed times served by the same source and underlying architecture, but this is a technically challenging issue and was considered beyond scope and budget of S-EUROGRID. The S-EUROGRID demonstration system with its two service streams is illustrated in Fig. 2. The S-EUROGRID system is not fully public but has been presented to a group of selected test users (e.g., the European Environment Agency (EEA), the BALTEX programme 


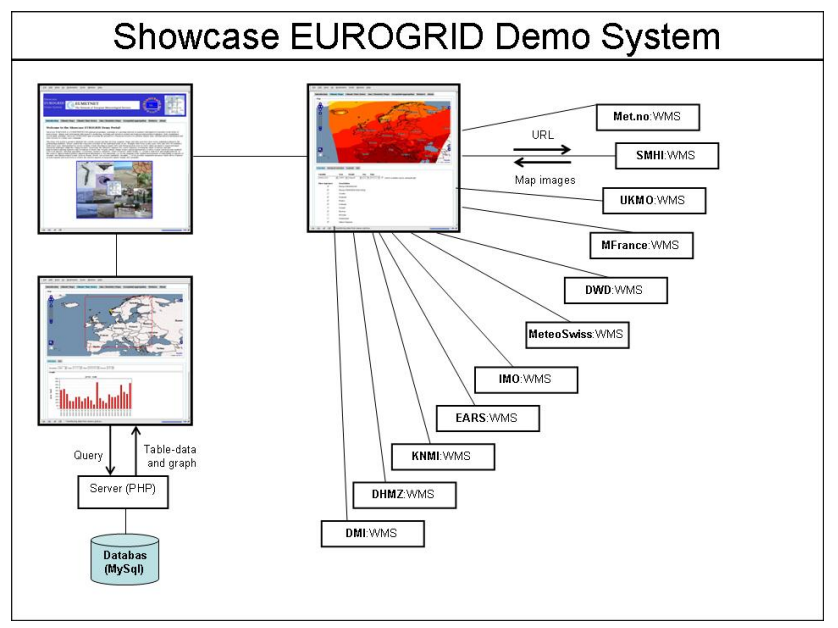

Figure 2. S-EUROGRID demonstration system overview. Two different service streams are shown: The right hand side illustrates the visualization of gridded data through the OpenLayers frontend and the underlying architecture of (distributed) OGC-based climate monitoring service components. The left part illustrates the access to time series for individual grid points through the OpenLayers map interface.

or the University of Lund, Sweden) who could and did provide feedback on the general idea.

\section{Example applications of Showcase EUROGRID services}

After entering the S-EUROGRID portal, the user can select between different applications. In addition to the selection of maps of ECVs, their visualization in the OpenLayers map service or the download of maps (OGC WMS) or raster data (OGC WCS), time series for specific locations can be extracted by mouse click. Furthermore, examples of integrated products combining atmospheric climate monitoring information with environmental information are available as well as examples of geospatially and temporally aggregated variables (e.g. long-term mean of temperature aggregated by country).

The following two sub-sections illustrate the potential of a mature EUROGRID system by the application of the SEUROGRID demonstration services to two example cases: The case of the European flooding in 2002 and the use of ECVs in combination with atmospheric chemistry data.

\subsection{Example 1: The flooding in Central Europe in 2002}

In August 2002, significant parts of Central Europe were hit by major flood events, including flash floods of smaller rivers and, subsequently, unprecedented floods of several larger rivers. The flood events were associated with two major record-breaking periods of rainfall (6-7 August and

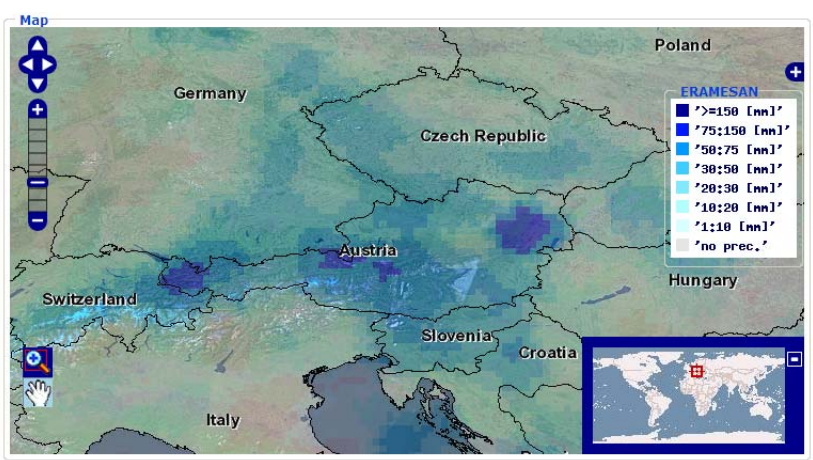

Figure 3. Snapshot from the S-EUROGRID demonstration site: Precipitation in Central Europe on 11-12 August 2002 (06:0006:00 UTC) on top of NASA global mosaic WMS-layer. The precipitation layer shown is based on the ERAMESAN dataset (Janson et al., 2007).

11-13 August 2002). These two periods of large-scale rainfall were preceded by a major period of convective-scale rainfall over Northern Germany in the first five days of August 2002. A detailed description of the precipitation events during the first half of August 2002 is provided by Deutscher Wetterdienst (2002) and Ulbrich et al. (2003a). The meteorological situation that caused this particular event is typical for many other large-scale flooding events in Central Europe (e.g. the major flood of 1997) and known as "Vb-weather situation" (Ulbrich et al., 2003b). The enormous amounts of rainfall during August 2002 caused a disastrous flooding of major rivers such as Elbe, Danube and Vltava. The list of dramatic consequences is long and starts with an estimated number of 100 lost lives, several hundred thousands of evacuated people and an estimated economical damage exceeding 15 billions of Euro (Swiss Re, 2002).

The case of the flooding of August 2002 can easily be illustrated using the S-EUROGRID demonstration data and visualization tools readily available to the test-user community. This is illustrated in Fig. 3 which is a snapshot from the SEUROGRID site showing a dynamically generated precipitation map covering the period 11 August 2002, 06:00 UTC to 12 August 2002, 06:00 UTC. The severity of the event is obvious with large daily sums of precipitation mainly over Austria on that particular day.

In addition to the description of the event with SEUROGRID precipitation maps, the user can also select time series by mouse click from the example database. This is demonstrated in Fig. 4, where a grid point close to the Austrian station Kremsmünster was selected. August 2002 is clearly identifiable as an outstanding month with a precipitation sum of $377 \mathrm{~mm}$ (slightly larger than the observed monthly sum of $350 \mathrm{~mm}$ at Kremsmünster for August 2002). A thorough evaluation and intercomparison of individual datasets is outside the scope of S-EUROGRID and is left for a potential follow-up work. Instead, this paper, focuses on 


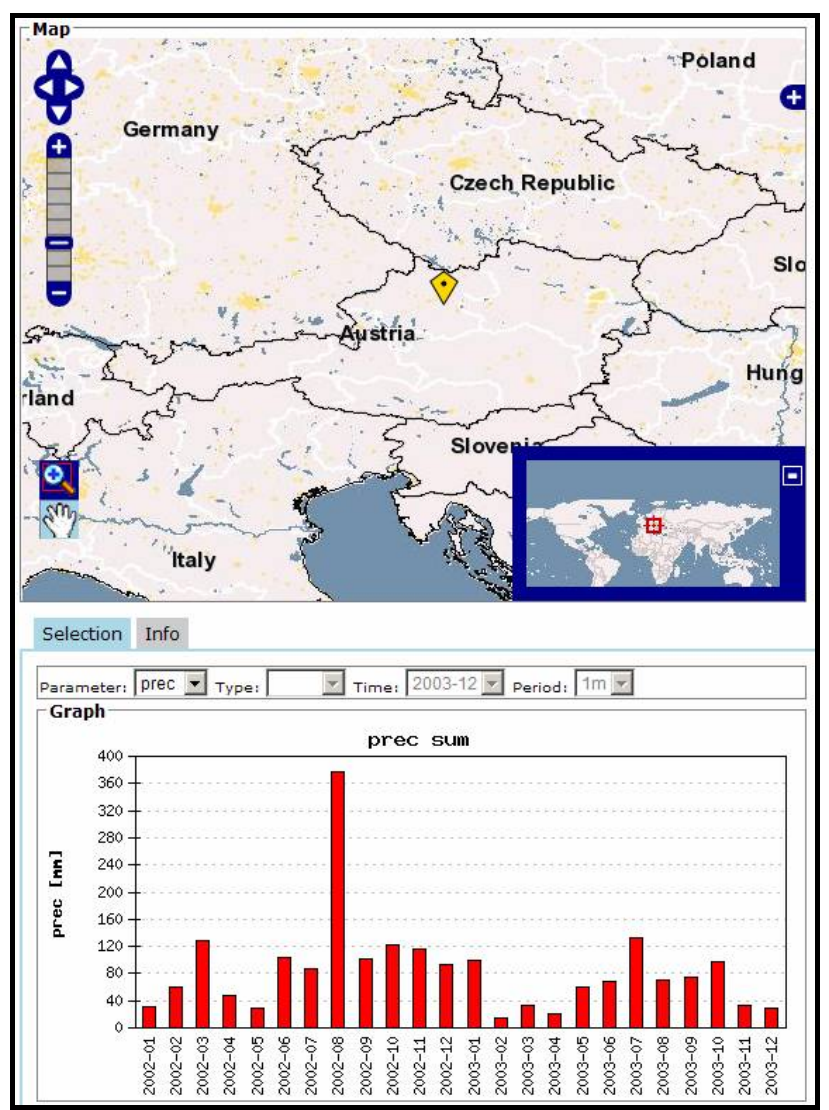

Figure 4. Example of a time series based on gridded precipitation data at a grid point close to observation site Kremsmünster $\left(14.13^{\circ} \mathrm{E}, 48.05^{\circ} \mathrm{N}\right)$, extracted from the S-EUROGRID demonstration database by mouse click.

a description of the EUROGRID idea. While Figs. 3 and 4 illustrate this idea and show the usefulness of EUROGRID's potential climate information services, they also underline the need for even better and more detailed pan-European gridded data of climate quality. The material currently available within S-EUROGRID is still very limited and its highresolution datasets - though very useful at a national level are not directly applicable for pan-European problems such as the flooding in August 2002 due to both differences in methodologies and data gaps (not every country has a national high-resolution dataset). The need to extend the SEUROGRID demonstration material with more data and, in particular, the preparation of consistent pan-European highresolution gridded data will have to be tackled by follow-up initiatives.

The S-EUROGRID portal does not offer server-side processing of the hosted data, but it is straight forward for the test-user to perform its own tailoring of information as a result of S-EUROGRID's architecture built around OGCservices. With the current technical solution a user can easily download data (using OGC WCS) directly into a GIS and perform aggregations in time (e.g. over the precipitation period in August 2002) and in space (e.g. over a catchment area affected by precipitation), which then could yield input to hydrological applications and analyses. This possibility of seamless user-side product tailoring is a very useful alternative to server-side product generation and is demonstrated in the next subsection.

\subsection{Example 2: Climate monitoring information and at- mospheric chemistry}

This example illustrates the cross-thematic application of climate monitoring information. Interoperability of services building on accepted standards is a pre-requirement to foster advanced usage of information and client-side post-treatment of information, e.g. to produce tailored products within the same thematic area but also across different thematic domains. This is illustrated here by the combined usage of atmospheric climate monitoring and atmospheric chemistry information.

In the atmospheric chemistry example presented here, precipitation from S-EUROGRID is used together with the analysed (gridded) concentration of pollutants in precipitation (based on measurements in Scandinavia). Download (WCS) of S-EUROGRID gridded precipitation and a simple clientside (GIS) multiplication with the analysed concentration in precipitation yields the wet deposition of a pollutant. This is illustrated in Fig. 5, where the monthly accumulated wet deposition of Sodium in Scandinavia is shown for January 2002. The pattern of wet deposition of Sodium in Fig. 5 is characteristic for winter months. Deposition values are generally small over land and large over the sea, especially over the North Sea. In particular, a pronounced gradient over the coastal areas is visible. This deposition pattern can be directly explained by seasalt which is the major source of Sodium. The seasalt contribution to the wet deposition explains both the coastal gradients and the regional gradient from the Baltic, Kattegatt and Skagerack towards the North Sea. The latter is directly coupled to the gradient in sea water salinity. This pattern is especially pronounced in months with strong westerly winds, e.g. winter and autumn, but normally less evident in e.g. the summer months.

\section{Conclusions}

S-EUROGRID had the objective to illustrate the idea of a European service for gridded climatological information and products, the EUROGRID. Using open standards and technologies (OGC) a demonstration system was built, providing example services to a community of test users. The architectural choice of OGC services turned out to be a good decision, allowing for the seamless integration of distributed information sources into the S-EUROGRID portal and providing an elegant way to disseminate both maps and data to 


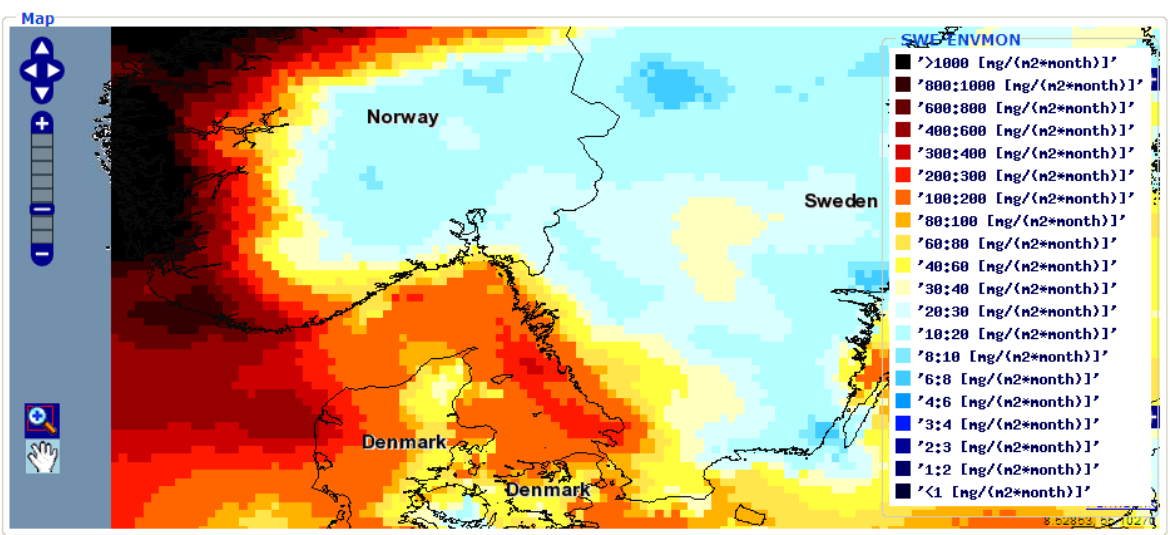

Figure 5. Example of an S-EUROGRID product derived by client-side tailoring (multiplication of S-EUROGRID precipitation with analysed species concentration in precipitation): Monthly sum of wet deposition of sodium in January 2002.

the test-user community. The seamless integration of information content with the GIS-environments of users directly facilitates further client-side processing and tailoring of information. Case studies from the flooding in Central Europe in August 2002 and a cross-domain application combining climate monitoring information with atmospheric chemistry show the usefulness and potential of the EUROGRID idea.

Results from test-user feedback so far confirm the need for European gridded climate monitoring information services and products, disseminated by state-of-the-art web services and technologies. However, they also indicate the necessity to move from a demonstration system and case studies to a mature and accessible service based on multi-decadal highresolution gridded data on ECVs. Follow-up initiatives of S-EUROGRID will need to take this feedback into account.

Acknowledgements. S-EUROGRID is a joint project between 16 countries within EUMETNET. Its tasks would not have been possible without the dedicated support of its partners and in-kind provisions of both work and data to the project.

Edited by: S. Szalai

Reviewed by: two anonymous referees

\section{References}

Deutscher Wetterdienst: Extreme precipitation amounts in Central Europe from 1-13 August, available at: http://www.meteo.uni-koeln.de/content/downloads/ rr_extreme200208_germany.pdf, 2002.

Jansson, A., Persson, C., and G. Strandberg: 2D meso-scale reanalysis of precipitation, temperature and wind over Europe ERAMESAN. Time period 1980-2004, SMHI, RMK 112, 2007.

OGC: www.opengeospatial.org, last access: 15 December 2008.

OpenLayers: www.openlayers.org, last access: 15 December 2008.

SwissRe, Floods are insurable!, available at: http://www.swissre. com/resources/5e8fa700462fa8c48348d3300190b89f-Floods_ en.pdf, 2002.

Ulbrich, U., Brücher, T., Fink, A. H., Leckebusch, G. C., Krüger, A., and Pinto, J. G.: The central European floods of August 2002: Part 1 - Rainfall periods and flood development, Weather, 58, 371-377, 2003a.

Ulbrich, U., Brücher, T., Fink, A. H., Leckebusch, G. C., Krüger, A., and Pinto, J. G.: The central European floods of August 2002: Part 2 - Synoptic causes and considerations with respect to climatic change, Weather, 58, 434-442, 2003b. 